\title{
Impacto de la pandemia COVID-19 en la investigación y docencia en las nanociencias y la nanotecnología en México
}

\section{Impact of the COVID-19 pandemic on research and teaching in nanosciences and nanotechnology in Mexico}

\author{
Gian Carlo Delgado Ramos, ${ }^{1, *}$ Rodolfo Zanella, ${ }^{2}$ \\ Leonel Cota Araiza, ${ }^{3}$ Rogelio López Torres ${ }^{1}$
}

\begin{abstract}
Since Mexico began to take measures against the COVID-19 pandemic in March 2020, the social, economic and political impacts and their implications for research, development and teaching in multiple areas of knowledge have diversified and deepened. Nanosciences and nanotechnology $(\mathrm{N} \& \mathrm{~N})$ are certainly no exception. This paper presents the main outcomes of a broad survey carried out among researchers in the country working in some area of $\mathrm{N} \& \mathrm{~N}$, which represents a third of said community. It analyzes the conditions of work and daily life in confinement, the productivity and development of research projects, institutional support, access to funds and financial management, and the impacts on the qualification of highly qualified personnel and on teaching. It is concluded that, in general terms, the delay in the research and qualification of that can be however a good qualified personnel in $\mathrm{N} \& \mathrm{~N}$ is located, at least, in the range of 2 to 3 years, a delay that can be a good opportunity to promote better practices of research, teaching, institutional administration and management that allow building a desirable resilience in the scientificeducational activity of the country, both in the $\mathrm{N} \& \mathrm{~N}$ field and in other areas.
\end{abstract}

KEYWORDS: COVID-19, lockdown, nanosciences, nanotechnology, Mexico.

RESUMEN: Desde que en México se comenzaron a tomar medidas ante la pandemia COVID-19 en marzo de 2020, los impactos sociales, económicos y políticos, y sus implicaciones en la investigación, desarrollo y docencia, en múltiples áreas del conocimiento se han diversificado y ahondado. Las nanociencias y la nanotecnología (NyN) ciertamente no son la excepción. Este artículo presenta los principales resultados de una amplia encuesta realizada a investigadores e investigadoras del país trabajando en algún área de las NyN, la cual representa una tercera parte de dicha comunidad. Analiza las condiciones de trabajo y vida cotidiana en confinamiento, la productividad y desarrollo de proyectos de investigación, el apoyo institucional, acceso a fondos y la gestión de financiamiento, y los impactos en la titulación de personal altamente calificado y en la docencia. Se concluye que, en términos generales, el retraso en la investigación y titulación de personal altamente calificado en NyN se ubica, por lo menos, en el rango de 2 a 3 años, un

Recibido: 3 de mayo 2021.

Aceptado: 27 de mayo 2021.

${ }^{1}$ Universidad Nacional Autónoma de México (UNAM), Centro de Investigaciones Interdisciplinarias en Ciencias y Humanidades.

2 UNAM, Instituto de Ciencias Aplicadas y Tecnología.

${ }^{3}$ UNAM, Centro de Nanociencias y Nanotecnología.

*Autor de correspondencia: giandelgado@unam.mx 
retraso que puede ser, sin embargo, una buena oportunidad para propiciar mejores prácticas de investigación, docencia, administración y gestión institucional que permitan construir una resiliencia deseable en la actividad científica-educativa del país, tanto en el ámbito de las NyN, como en otras áreas.

PALABRAS CLAVE: COVID-19, confinamiento, nanociencias, nanotecnología, México.

\section{Introducción}

Desde que se notificara el 30 de diciembre de 2019 sobre la aparición de una neumonía de causa desconocida en Wuhan, China, los impactos a la salud y a la vida de millones de personas son patentes. Hasta mayo de 2021, se reportaban 166.8 millones de casos de contagio por SARS-CoV-2 confirmados a nivel global, y casi 3.5 millones de defunciones - aunque se cree que la cifra real podría ascender a 8 millones-. Los casos confirmados en México hasta mediados de mayo de 2021 sumaban 2.39 millones, con más de 221 mil defunciones por COVID-19 oficialmente reconocidas. Los estados de la república con mayor número de casos hasta ese momento eran la Ciudad de México, Baja California y Querétaro (tabla 1).

A pesar de los notorios avances en el estudio del virus SARS-CoV-2 que, entre otras cuestiones, han posibilitado el desarrollo de diversas variantes de vacunas, es de subrayarse que aún persiste la duda sobre su origen. Si bien la Organización Mundial de la Salud se inclina más por su un origen zoonótico, es decir, derivado de una infección naturalmente transmisible de animales vertebrados a humanos (OMS, 2021), la falta de pruebas contundentes sobre tal presunción hace que estrictamente siga siendo también válida la hipótesis sobre la liberación accidental del coronavirus desde algún laboratorio (Bloom et al., 2021). En todo caso, lo que es un hecho es que se trata de una enfermedad infecciosa emergente (Haider et al., 2020) que ha y sigue trastocado múltiples dimensiones de la vida humana, incluyendo la económica, política y cultural, la producción de conocimiento y la innovación tecnológica, y muchas otras actividades de la vida social.

El grueso de las economías se encuentra en franca recesión con una contracción de la economía mundial de alrededor del 3.3\% del PIB (FMI, 2021), siendo China la única economía de entre las más grandes que logró un crecimiento de $2.3 \%$ en 2020 (NBSC, 2021). Los niveles de desempleo son crecientes en muchos países, sobre todo del Sur Global y con mayores afectaciones a mujeres, jóvenes y personas con experiencia limitada (FMI, 2021). Sectores económicos enteros afrontan una parálisis o retos mayores, particularmente el sector turismo (UNWTO, 2020). El sector más beneficiado ha sido sin duda el farmacéutico con ganancias millonarias (tan solo Moderna espera ganancias por 19.6 mil millones de dólares en 2021, 12.2 mil millones en 2022 y 11.4 mil millones en 2023, mientras que Pfizer estima ganancias por 15 a 30 mil millones de dólares tan solo para 2021; (Kollewe, 2021)). A pesar del pujante mercado que se gesta en medio de la adversidad global, es 
Tabla 1. Casos confirmados de COVID-19 en México hasta mayo de 2021 (diez primeros lugares a nivel estatal).

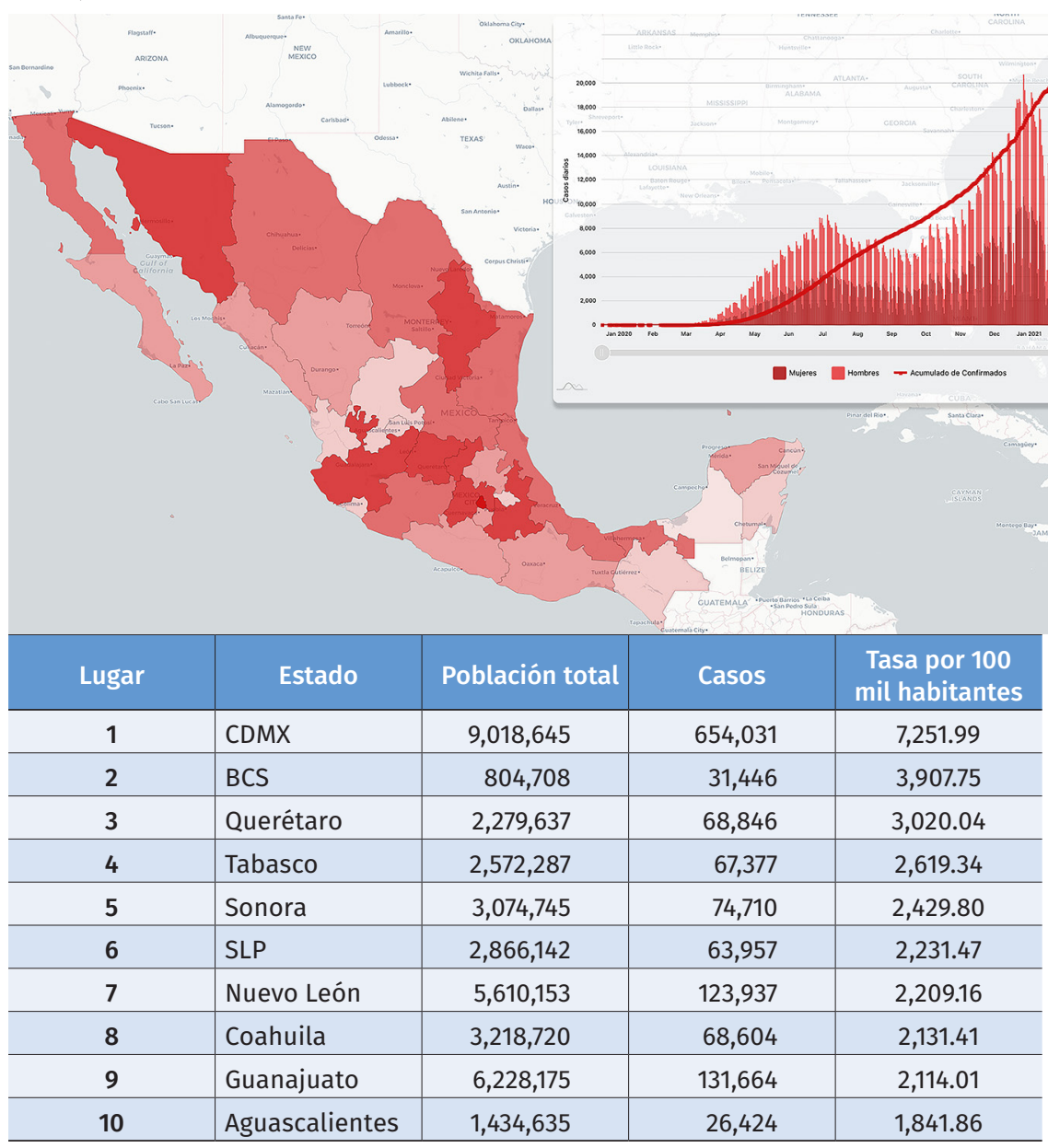

Fuente: Tomado de datos.covid-19.conacyt.mx el 20 de mayo de 2021.

claro que falta camino por andar y diversos retos por resolver, no únicamente en materia de tasas mayores y más balanceadas de vacunación (el Norte Global presenta mayores tasas de vacunación que el Sur Global resultando en una apropiación de alrededor del $75 \%$ de las vacunas por parte de apenas 10 países (tabla 2)), sino incluso de innovación para la prevención, diagnóstico y tratamiento de la enfermedad causada por el virus SARSCoV-2, la cual no acaba de ser entendida del todo a pesar de tratarse de una enfermedad que afecta principalmente, pero no únicamente, los sistemas respiratorio y circulatorio (Chung et al., 2021; Chilazi et al., 2021; AduAmankwaah, 2020). 
Tabla 2. Tasas de vacunación en países selectos hasta mayo de 2021 (\# de habitantes por cada cien).

\begin{tabular}{l|c|l|c}
\hline País & $\begin{array}{c}\text { Tasa de } \\
\text { vacunación }\end{array}$ & País & $\begin{array}{c}\text { Tasa de } \\
\text { vacunación }\end{array}$ \\
\hline Estados Unidos & 85.55 & Rusia & 16.4 \\
\hline Reino Unido & 84 & Australia & 12.11 \\
\hline Alemania & 48.3 & Brasil & 25.93 \\
\hline Francia & 43.5 & Argentina & 23.8 \\
\hline Italia & 46.1 & México & 19.38 \\
\hline Japón & 4.42 & Venezuela & 0.81 \\
\hline India & 13.46 & Egipto & 1.92 \\
\hline China & 26.39 & R.D. Congo & 0.22 \\
\hline
\end{tabular}

Nota: Hasta mayo de 2021 más de 700 millones de personas habian recibido una o dos dosis. Fuente: Elaboración de los autores con base en datos del 24 de mayo de 2021 de la OMS (https:// covid19.who.int).

El papel que en este contexto pueden tener la innovación y el desarrollo, incluyendo la $\mathrm{NyN}$, es crucial, sobre todo de cara a las fuertes asimetrías imperantes tanto en el acceso a vacunas, tratamientos y equipo médico, como en general a servicios médicos y otros servicios cruciales para la prevención de la transmisión del virus SARS-CoV-2 tales como el de agua y saneamiento (Lancet, 2021; IFC, 2020; Delgado y López, 2020). La dependencia a la importación de ventiladores y equipo protector en medio de una urgencia global, y ahora a la importación de vacunas, pone en una posición endeble al país que, hasta abril de 2021, había gastado 15.8 mil millones de pesos en contratos para la adquisición de vacunas, casi el equivalente al $60 \%$ del presupuesto del Consejo Nacional de Ciencia y Tecnología (Conacyt) para 2021.

\section{Breve panorámica del rol de la NyN en la lucha contra la COVID-19}

La nanotecnología ha sido importante en la lucha contra la COVID-19 y se considera que jugará un papel cada vez más relevante tanto en la prevención y el diagnóstico, como en la mejora de sistemas de entrega de agentes antivirales y el desarrollo de estos últimos de manera que puedan ser específicamente diseñados contra el SARS-CoV-2, contexto en el que se han señalado como promisorios los materiales inorgánicos autoensamblados y las nanopartículas de base péptida (Rashidzadeh et al., 2021), así como la nanoformulación de drogas como el corticosteroide dexametasona (Lammers et al., 2020).

Otras aplicaciones son el uso de nanomateriales para máscaras protectoras y desinfectantes virales efectivos, por ejemplo, basados en nanopartículas metálicas (Talebian et al., 2020) o nanobiomoléculas (caso de nbelyax desarrollada por Gresmex, usada en cubrebocas y gel; UAM, 2018). Igual- 
mente, la nanotecnología puede jugar un rol importante en el desarrollo de sistemas de diagnóstico rápido y preciso que puedan mejorar la técnica hasta ahora usada de reacción en cadena de la polimerasa (PCR) que se basa en la detección del material genético del virus, pero que demanda varios pasos de purificación, lo que supone ser un reto para la obtención sistemática de resultados confiables dado que las impurezas pueden afectar la detección del virus. Como alternativa se explora el uso de nanopartículas de oro (Pramanik et al., 2021; Ventura et al., 2020), grafeno (Seo et al., 2020), estructuras metal-orgánicas (Rabiee et al., 2020) o nanotubos de dióxido de titanio funcionalizados con titanio (Vadlamani et al., 2020).

Otra aplicación es el desarrollo de vacunas que hacen uso de nanomateriales para la entrega más efectiva de antígenos a células $\mathrm{T}$ y $\mathrm{B}$, tales como las vacunas mRNA, es decir, aquellas que usan el ARN mensajero para introducir secuencias genéticas específicas de una proteína viral en el cuerpo (Florindo et al., 2020; Pardi et al., 2018). A diferencia de las vacunas tradicionales que introducen virus atenuados o inactivos en el cuerpo, las vacunas de Pfizer-BioNTech y Moderna son encapsuladas en nanopartículas lipídicas cargadas positivamente lo que les permite ser más resistentes a la degradación ribonucleica (Milane y Amiji, 2021; MHRA, 2020). Pese a ello, más investigación es necesaria pues la efectividad de esas vacunas ha sido cuestionada ante la emergencia de nuevas variantes del SARS-CoV-2 como las de Sudáfrica y Brasil (Neidleman et al., 2021). Cabe precisar que este tipo de tecnología puede ser efectiva en otras enfermedades, incluyendo las autoinmunes, por lo que hay un doble incentivo en su avance (Velikova y Georgiev, 2021; Florindo et al., 2020; Marc, Domínguez y Gamazo, 2015).

La investigación en NyN en México está buscando también dar respuesta a la pandemia COVID-19 (Chávez et al., 2020; Durán et al., 2020; de la Torre et al., 2020; véase también más adelante), más allá de otras áreas como las que incluso han llevado a la innovación en ventiladores de emergencia, el VSZ20-2, el Ehécatl T4 y el Gätsi (Navarrete y Treviño, 2020; Rodríguez, 2020). A pesar de ello, el quehacer científico en todos los campos de conocimiento, incluyendo la propia NyN, está siendo impactado por la pandemia COVID-19 y las medidas tomadas para afrontarla. Para evaluar tal fenómeno, desde Mundo Nano. Revista Interdisciplinaria en Nanociencias y Nanotecnología se procedió a realizar una encuesta a la comunidad NyN en México. Las características de la muestra encuestada y los principales resultados obtenidos se presentan a continuación.

\section{Características de la muestra}

Durante los meses de febrero y marzo de 2021 se realizó una encuesta virtual a la comunidad de académicos de diversas universidades públicas y privadas, centros de investigación públicos y a una empresa de México cuya línea de investigación o actividad se centra en alguna de las diversas áreas de 
las nanociencias y la nanotecnología. De un universo estimado en alrededor de 450 investigadores e investigadoras de unas 56 instituciones (CIMAV, 2008), se recibieron 131 respuestas de 34 entidades de investigación y una empresa. La muestra representa el $29 \%$ de la comunidad científica y el $60 \%$ de las instituciones realizando investigación en $\mathrm{NyN}$ si se considera que la mencionada estimación sigue siendo válida. Los encuestados corresponden a 15 de los 32 estados de la república, tal y como se presenta en la figura 1.

Figura 1. Estados con por lo menos una encuesta aplicada.

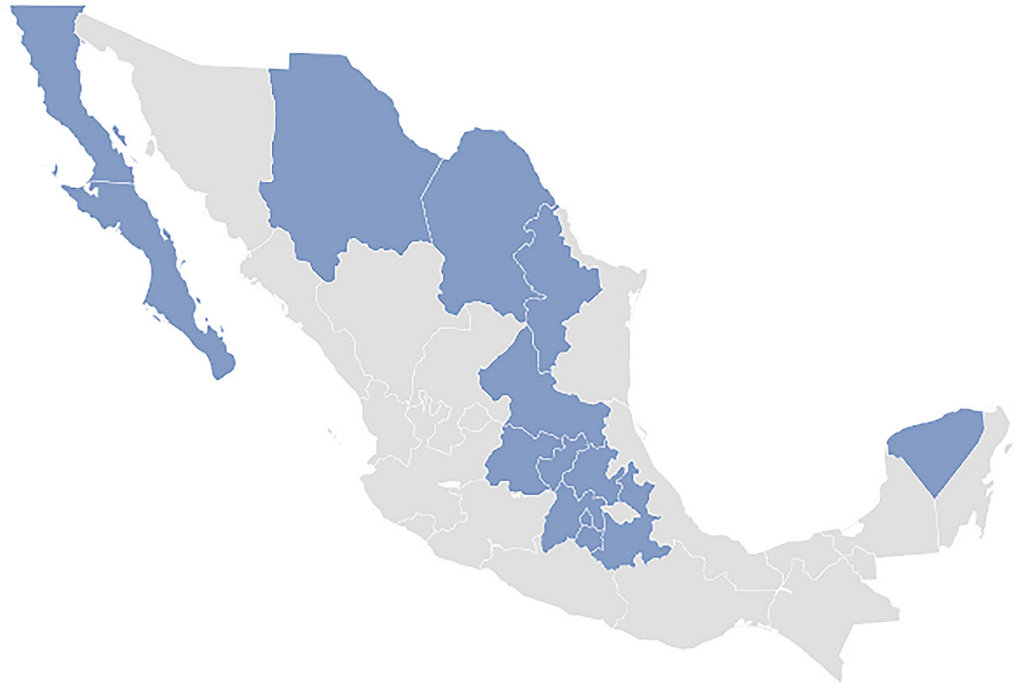

Fuente: Elaboración de los autores.

La muestra de encuestados es relativamente equilibrada en términos de edad y género, tal y como se constata en las figuras 2A y 2B. El $85.5 \%$ de los académicos y académicas encuestados pertenecen al Sistema Nacional de Investigadores del Conacyt. Además, algunos también son integrantes de la Academia Mexicana de Ciencias (21.4\%) o de alguna otra academia nacional o internacional (39\%).

La mayoría de la investigación que realizan las y los encuestados recibe financiamiento de su propia entidad o de parte del Conacyt, siendo marcadamente menor el apoyo por parte de otros organismos federales, gobiernos estatales o iniciativa privada (figura 3 ).

El tipo de investigación de los y las encuestadas son de tipo experimental (73.5\%), seguido por las de carácter teórico (16\%), documental (8\%) y de otra naturaleza (2.5\%). Las líneas de investigación son principalmente sobre nanopartículas, bionanopartículas, películas delgadas, polímeros y otros nanomateriales en aplicaciones que van desde la agricultura, la generación de 
Figura 2. Edades y género de los y las académicas encuestadas.

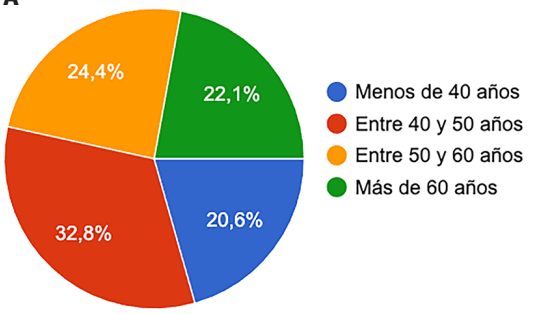

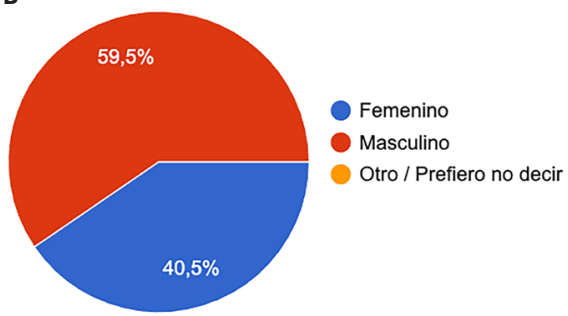

Fuente: Elaboración de los autores.

Figura 3. Financiamiento de la investigación realizada por los y las encuestadas.

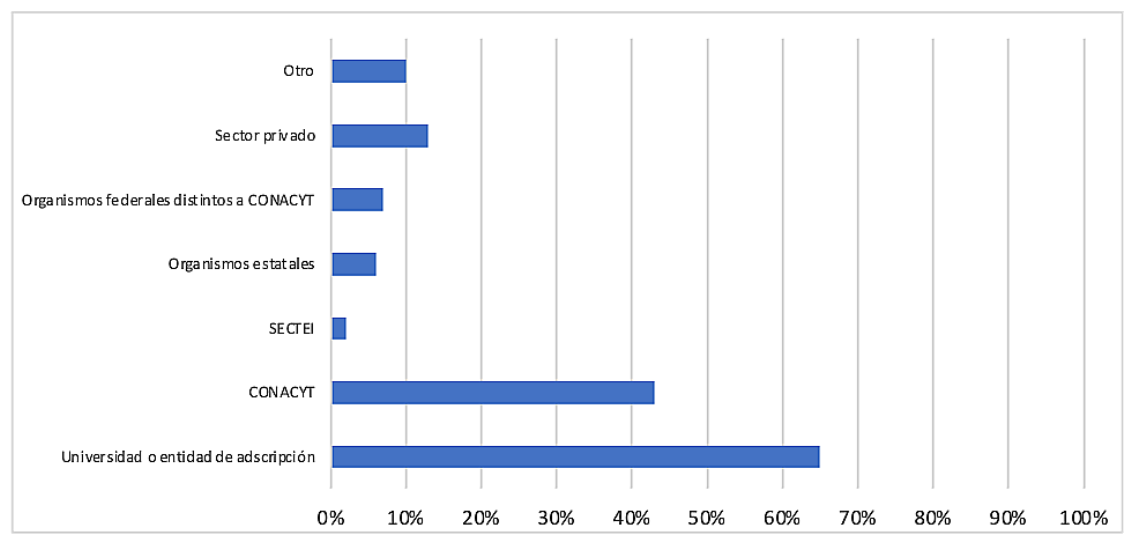

Fuente: Elaboración de los autores.

energía, y el desarrollo de semiconductores, dispositivos y circuitos electrónicos, hasta su aplicación en el ámbito de la salud. Se suman otras líneas de investigación en nanofotónica, cristalografía, nanocatálisis, química computacional, prevención y remediación ambiental, nano-toxicología, educación y aspectos sociales de la $\mathrm{NyN}$, entre otras.

Son destacables, desde la perspectiva de este trabajo, algunas líneas de investigación reportadas con potencial incidencia directa o indirecta en la prevención, detección o contención del SARS-CoV-2 tales como:

- Plasmónica con potencial aplicación en el diagnóstico viral, incluyendo el desarrollo de sensores plasmónicos de COVID-19.

- Nanopartículas o nanomateriales compuestos con actividad antiviral y antibacteriana.

- Funcionalización de mascarillas de protección con nanopartículas.

- Aplicación de nanopartículas de plata Argovit como medio preventivo contra COVID-19. 
- Desarrollo de materiales de referencia para la detección de SARSCoV2.

- Nanocompuestos cerámicos y poliméricos con aplicaciones en medicina terapéutica y diagnóstica.

- Nanopartículas tipo virus (VLP’s, por sus siglas en inglés) para el transporte de fármacos.

\section{Resultados}

La encuesta se estructuró en torno a los impactos de la pandemia en: a) las condiciones de trabajo y vida cotidiana en confinamiento; b) la productividad y desarrollo de proyectos de investigación; c) el apoyo institucional, acceso a fondos y la gestión de financiamiento, y, d) en la titulación de personal altamente calificado y docencia. Asimismo, se indagó en los retos institucionales derivados de la pandemia COVID-19 y las medidas tomadas para su control.

\section{(a) Condiciones de trabajo y vida cotidiana en confinamiento}

Como se puede observar en la figura 4A, poco menos de la mitad (42.9\%) de los y las encuestados advierten que la situación de confinamiento ha significado un mayor tiempo dedicado a hijos o dependientes, aunque una proporción similar (39.3\%) precisa que el confinamiento no ha representado una mayor dedicación de tiempo a sus hijos y dependientes. De manera muy similar, casi la mitad de los encuestados han experimentado una situación de mayor tiempo invertido en la limpieza y mantenimiento del hogar, y una proporción parecida comunica que eso no ha sido el caso. Finalmente, $7 \%$ advirte que otra persona se hace cargo de ello (figura 4B). Una encuestada reporta su preocupación por el limitado tiempo que logra tener para poder hacer investigación y asistir a su laboratorio debido a la atención que requieren sus hijos dado que no pueden asistir a la escuela en tiempos de confinamiento.

Figura 4. Grado de afectación al trabajo académico por el incremento en el tiempo dedicado al cuidado de hijos, dependientes y del hogar.

A. Grado de afectación en el tiempo dedicado a hijos y dependientes.

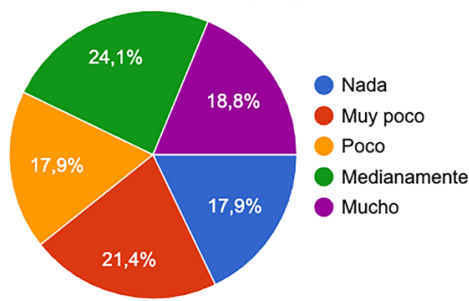

Fuente: Elaboración de los autores.
B. Grado de afectación en el tiempo dedicado al hogar.

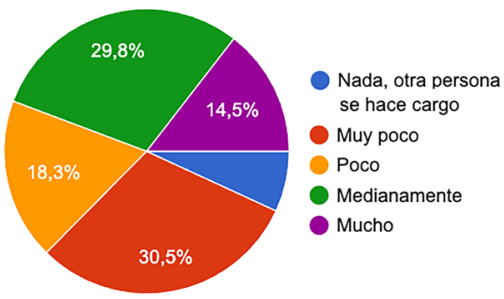


(b) Impactos de la pandemia COVID-19 en la productividad y desarrollo de proyectos de investigación

En general, la productividad de las y los encuestados se ha visto afectada. El $42 \%$ reporta una afectación media mientras que $29 \%$ una afectación alta; el $29 \%$ restante precisa una afectación baja, muy baja o ninguna afectación. La principal razón es el limitado o nulo acceso a laboratorios para el $84.7 \%$ de los casos, ello seguido de limitaciones de tiempo en condiciones de confinamiento y por la dificultad de efectuar trabajo colaborativo $(64.1 \%$ y $55 \%$ de los casos, respectivamente) (figura 5).

Figura 5. Tipo e intensidad de impactos del confinamiento por COVID-19 en la investigación en NyN.

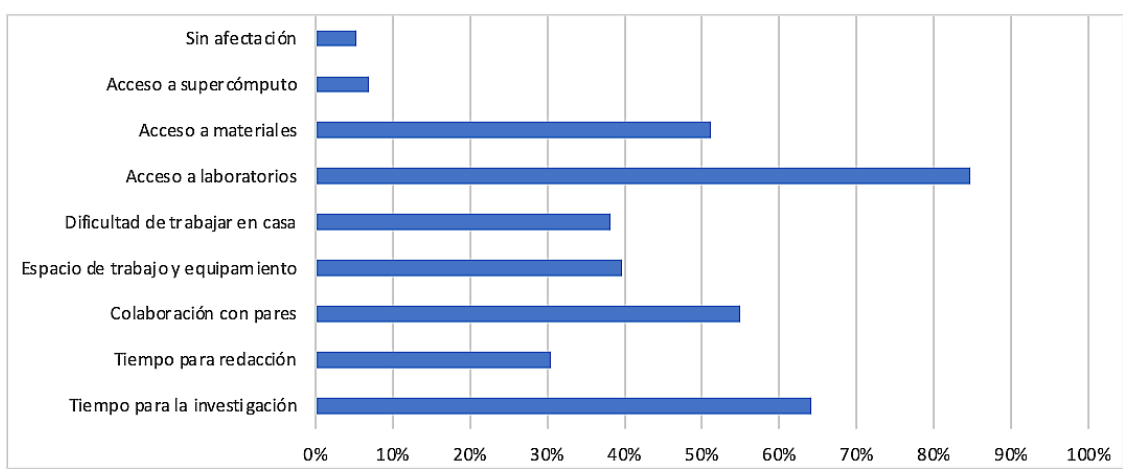

Fuente: Elaboración de los autores.

Cabe precisar que en los casos en que los o las encuestadas tienen posibilidad de usar las instalaciones de su entidad de adscripción, las medidas de prevención reportadas son esencialmente el uso de gel y cubrebocas, así como el requerimiento de sana distancia. En menor medida se reporta la instalación de un filtro sanitario de ingreso.

\section{(c) Apoyo institucional, acceso a fondos y gestión de financiamiento}

La contracción económica que la pandemia COVID-19 ha provocado (en México de alrededor del 8.5\% del PIB durante 2020 (INEGI, 2021)) se ha traducido en una reorientación del presupuesto público y una reducción del financiamiento internacional y privado, particularmente en áreas no dedicadas directamente a la prevención, diagnóstico y tratamiento de la enfermedad causada por el virus SARS-CoV2. De cara a tal situación, la gran mayoría de las y los encuestados informan dificultades en el acceso a fondos para continuar proyectos o para desarrollar nuevos (figura 6). Por un lado, en el caso del financiamiento público, las convocatorias nacionales e internacionales se han atrasado y los resultados se retrasan con frecuencia. Las gestiones para acceder a los re- 
Figura 6. Dificultad en el acceso a fondos para continuar proyectos o desarrollar nuevos en tiempos de COVID-19.

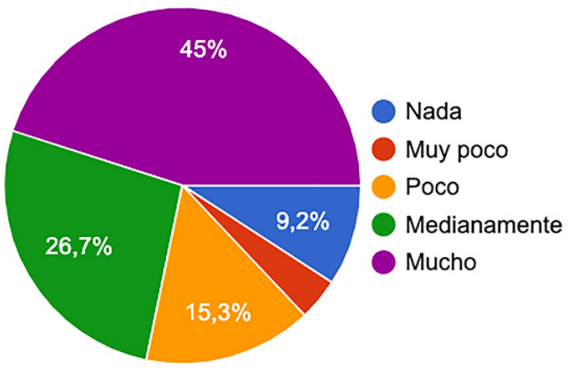

Fuente: Elaboración de los autores.

cursos se han visto afectadas sea por contratiempos en las entidades de gobierno financiadoras o por limitaciones de personal y lentitud de los procesos de las propias universidades receptoras de los recursos. En lo que respecta al financiamiento privado, por un lado, se reporta que en general las empresas han detenido la inversión en investigación y desarrollo o bien han reorientado sus actividades y metas, ello como parte de las medidas de austeridad que han tomado. Por el otro, se informa la dificultad de continuar incidiendo en los nexos con el sector privado debido a que las instituciones de investigación están cerradas y porque las autoridades de estas laboran desde casa lo que dificulta toda interacción (situación aún válida hasta finales de mayo de 2021). Eso es doblemente cierto cuando se presentan situaciones similares en el ámbito productivo. En los casos en los que se logra continuar la vinculación con el sector privado, se reportan dificultades importantes para ejecutar los proyectos debido a la falta de personal, tal y como a continuación se precisa.

Adicionalmente a lo referente al financiamiento, y como se dijo, se constatan una serie de afectaciones en las gestiones administrativas y de otros servicios, lo que ha impactado la investigación y desarrollo tecnológico en diversas áreas de la NyN. Especialmente denotan cuatro afectaciones: mantenimiento de equipo y acceso a instalaciones lo reportan el $81.7 \%$ de los encuestados; limitaciones de personal para el 66.4\%; adquisición de materiales e insumos para el 52.7\%; y gestiones administrativas relacionadas con el uso de recursos y desarrollo de proyectos de investigación en el caso del $50.4 \%$ de los encuestados.

En general se señala el enlentecimiento de todos los procesos administrativos y de gestión, incluyendo el acceso a servicios analíticos (de caracterización de materiales y otros) dado que muchos técnicos de laboratorio no están laborando o bien porque se trabaja con el mínimo de personas posible. Es importante precisar que en el caso de algunos técnicos de laboratorio se han reportado limitaciones para laborar por ser parte de los grupos vulnerables y de alto riesgo, o porque deben cuidar a sus dependientes en casa debido al cierre de escuelas como parte de las medidas de confinamiento. 
También se reportan dificultades en el acceso y mantenimiento de equipo, así como en la adquisición de materiales o componentes de equipamiento de laboratorio (debido a los retrasos verificados en las fábricas y por ende en los proveedores, pero también porque los técnicos especializados del extranjero que dan servicio a ciertos equipos no tienen permitido viajar a México). Incluso, para los casos con investigación clínica, el acceso a muestras hospitalarias o de campo se ha retrasado, afectando la entrega en tiempo y forma de avances o resultados de investigación. Lo mismo aplica para el caso de experimentos con organismos vivos pues las normativas para evitar contagios han limitado ese tipo de trabajo.

Los retrasos en el trabajo experimental preocupan a la comunidad de sobre manera pues afecta tanto la evaluación de los investigadores como el proceso mismo de titulación y evaluación de programas de posgrado (véase más adelante). La cancelación de estancias por parte de las autoridades universitarias o de otras instituciones de investigación también ha impactado la posibilidad de realizar pruebas o para desarrollar investigación colaborativa con otras universidades, lo cual se traduce en importantes limitantes para algunas líneas de investigación en NyN. A tal escenario se suma la cancelación de numerosos congresos y de otras modalidades de encuentros académicos, mientras que otros han logrado transitar a modalidad virtual sin lograr reemplazar las bondades de la interacción presencial.

Entre los señalamientos recurrentes, además de los antes expuestos, están aquellos que refieren a la (in)flexibilidad por parte de entidades financiadoras y administrativos, un aspecto que presenta una valoración mixta. Por ejemplo, la tercera parte de los y las encuestadas reportan falta de total empatía y flexibilidad por parte de las instituciones y organismos financiadores, mientras que una cuarta parte indica que la empatía y flexibilidad es limitada o muy limitada (figura 7). Lo dicho se traduce, por ejemplo, en dificultades (sobrecarga administrativa o de gestión, particularmente en lo que respecta a fondos federales) para la solicitud de extensión de fechas para la entrega de re-

Figura 7. Grado de empatía y flexibilidad por parte de instituciones u organismos financiadores debido a las condiciones excepcionales en tiempos de COVID-19.

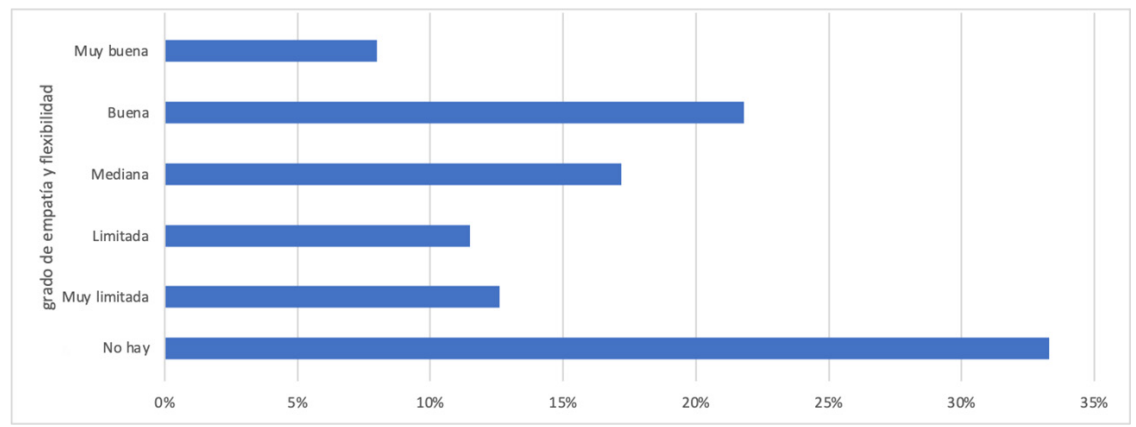

Fuente: Elaboración de los autores. 
sultados o de entregables parciales (incluyendo reportes financieros y de otra índole), así como en limitaciones para modificar rubros presupuestales (por ejemplo, transferencia de partidas, especialmente las que refieren a congresos, viáticos y pasajes), todo ello en un contexto de tiempos de respuesta a solicitudes extremadamente largos si se consideran los tiempos delimitados de los proyectos. Se ha incluso señalado que tal inflexibilidad de parte de algunas instituciones financiadoras se ha dado en medio de retrasos en los pagos de financiamiento y en el ajuste de los montos originalmente sugeridos para algunas convocatorias.

Por otro lado, también se constatan opiniones contrarias, es decir, casos diversos en los que se reportan medidas de flexibilización, particularmente en lo que respecta a los ajustes en los plazos de entrega de avances o resultados de investigación (caso de alrededor del $30 \%$ de los encuestados) y en la autorización en la transferencia de recursos (caso del $27.5 \%$ de los encuestados), modificación de los objetivos y metas de los proyectos (caso del $24.4 \%$ de los encuestados). En menor medida se reportan ajustes en las prácticas de administración y gestión de recursos o en el avance de mecanismos de buena comunicación para la resolución de problemas específicos (en ambos casos con menos del $20 \%$ de los encuestados).

La apreciación mixta antes descrita devela la existencia de condiciones diversas en las instituciones de investigación, sus gestores y administradores que se traducen en mejores o más limitadas condiciones para la investigación en NyN en un contexto de pandemia. Lo mismo aplica para el caso de las instituciones de financiamiento de gobierno donde pareciera que el reto es relativamente mayor dadas las dinámicas burocráticas y políticas que las caracterizan, por los cambios de prioridades y de personal, por ajustes presupuestales realizados, y por la propia afectación de su personal debido a la transmisión de la enfermedad por coronavirus, lo cual se ha traducido en tiempos operativos particularmente lentos.

En todo caso, lo que es consenso es la preocupación generalizada de la evaluación por parte del Sistema Nacional de Investigadores del Conacyt, tanto por las dificultades para realizar trabajo experimental y para titular a personal altamente calificado, como por el retraso de los procesos de muchas revistas científicas a causa de la pandemia. Los resultados logrados en 2020 en muchos de los casos ha sido producto de la inercia del trabajo pre-pandemia por lo que los mayores efectos se esperan sean observables en los informes de 2021. Un pronunciamiento de parte de Conacyt al respecto se considera deseable.

Desde la perspectiva de los directivos o responsables institucionales encuestados, se confirman una serie de situaciones, demandas y retos, destacando los siguientes: necesidad de adaptarse a nuevas condiciones lo que incluye dar respuesta a planes institucionales de reacción cambiantes y en ocasiones truncos; aplicación de reglamentos de emergencia sanitaria en medio de presiones para continuar con proyectos de investigación que requieren el uso de instalaciones; dificultad para mantener una fluida comunicación con el grueso 
de académicos y personal en general de sus instituciones; retos para garantizar la sanitización de espacios semicerrados y cerrados (sobre todo áreas especializadas con aire acondicionado) y falta de personal e insumos para hacerlo adecuadamente; imposibilidad o dificultad para habilitar el uso de laboratorios a la par de las mencionadas presiones para su uso, aún en semáforo epidemiológico en rojo; desafíos administrativos y de otra índole para gestionar adquisiciones y servicios en un contexto de restricciones presupuestales; contratiempos con personal administrativo para flexibilizar y sensibilizar procesos; rigidez de organismos financiadores externos en relación con procesos administrativos e informes de proyectos; vandalismo a instalaciones institucionales; así como el fallecimiento de personal a causa de la COVID-19. Todas estas situaciones ponen en doble desventaja a la comunidad científica mexicana abocada a las NyN pues se suman a las condiciones diferenciales que de por sí ya experimentaba antes de la pandemia COVID-19 (presupuestales, de infraestructura, etc.). Ante el avance de apoyos sensibles a los impactos de la pandemia COVID-19 en otras latitudes, la percepción mayoritaria de las y los encuestados es que los resultados de la investigación en $\mathrm{NyN}$ se han atrasado en un rango de 1 a 3 años, tal y como se muestra en la figura 8.

Figura 8. Retraso percibido en los resultados de investigación en NyN.

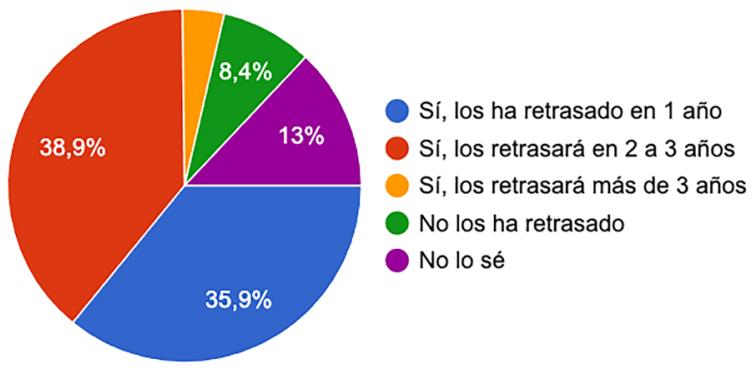

Fuente: Elaboración de los autores.

\section{(d) Titulación de personal altamente calificado y docencia}

El confinamiento por la pandemia COVID-19 ha afectado la docencia de modo sustancial. El 48.1\% de los y las encuestadas consideran que la calidad del aprendizaje se ha visto comprometida. El tránsito a modalidades virtuales de docencia ha demandado tiempo para replantear programas y dinámicas de clase, lo cual ha impactado en el tiempo disponible para la investigación. La curva de aprendizaje tanto por parte del profesorado como del alumnado para transitar de modalidades híbridas de aprendizaje presencial, que vinculan lo teórico con lo experimental, ha sido importante en el paso hacia modalidades virtuales de docencia prácticamente teóricas o con muy limitada experimentación. 
A tal escenario se suman las diversas situaciones que afrontan los alumnos, a quienes se les dificulta tener un espacio y conectividad apropiada para tomar clases en casa ( $83.2 \%$ de los encuestados reportan problemas de conectividad por parte de los alumnos y $41.2 \%$ la carencia de computadora personal en casa) y para enfocarse en el trabajo académico ( $49.6 \%$ de los encuestados informa que los alumnos afrontan esta situación). A eso se suman problemas de estabilidad personal y/o familiar especialmente relacionados con ingresos económicos (36.6\% de los encuestados lo confirman) o situaciones indeseables de salud (38.9\% de los encuestados informan casos de alumnos o de sus familiares contagiados del virus SARS-CoV-2). Todo en conjunto afecta el rendimiento académico del estudiantado y su estado anímico, además de impactar en múltiples ocasiones la disponibilidad de tiempo cuando se ven orillados a apoyar a la familia asumiendo responsabilidades adicionales en casa. No es casual que se reporte el desánimo en el desarrollo de trabajos de grado, pero aún con más frecuencia el abandono de estudios, particularmente a nivel licenciatura donde contar con beca es menos frecuente que en posgrado $y$, cuando es así, esas difícilmente son significativas en el ingreso personal o familiar (ello en comparación con las becas posgrado). Por lo anterior, no solo se reporta la falta de motivación, sino múltiples casos de depresión entre el alumnado.

Por su parte, el profesorado reporta en $21.4 \%$ de los casos problemas de conectividad para impartir adecuadamente sus clases, mientras que $26 \%$ considera que el tránsito hacia modalidades virtuales de enseñanza ha implicado un mayor tiempo destinado a la preparación de clases, lo cual como ya se dijo, compite con el tiempo de investigación y con el tiempo personal. En algunos casos las limitaciones comprenden la falta de software adecuado para la impartición de clases (caso del $4.6 \%$ de los encuestados) y la dificultad de su comprensión (caso del 7.6\% de los encuestados); contexto en el que cabe advertir que los softwares dominantes son Zoom y Classroom, seguidos por Meet, Microsoft Teams, BlueJeans, GotoMeeting, Blackboard, Miro, Edmodo, entre otros. Solo $27.5 \%$ de los y las encuestadas informan no haber visto afectada su actividad docente. El apoyo institucional para la adquisición de licencias y asesoramiento para la implementación de docencia virtual, en muchos casos ha sido limitado. Algunos académicos han tenido que aprender sobre la marcha, a la vez que se han visto obligados a sufragar individualmente los costos de software y de mejor conectividad. El resultado ha sido una migración lenta y desigual hacia la docencia virtual en muchas universidades públicas.

Desde luego no todo ha sido negativo. Se reportan experiencias de docencia virtual en NyN exitosas; una relativa (y en ciertos casos obligada) actualización del profesorado en el uso de herramientas tecnológicas de frontera para su práctica docente y de colaboración con pares; y un mayor alcance de algunos encuentros académicos realizados de manera virtual cuyos costos son radicalmente menores.

Ahora bien, en lo que respecta al acceso limitado a laboratorios cabe subrayar que ello ha impactado fuertemente a tesistas de posgrado, viendo dete- 
nido o entorpecido el trabajo experimental que suele ser crucial en sus trabajos de grado. En algunos casos se reportan restricciones de acceso a estudiantes provenientes de instituciones externas lo que afecta, como ya se precisó, a estudiantes realizando estancias de investigación o con necesidad de hacerlo (por ejemplo, para poder realizar pruebas de laboratorio especializadas). Derivado de ello se han dado procesos de restructuración o adaptación de los proyectos de investigación, sus objetivos y metas, lo que sin duda afecta cualitativamente el avance de la generación de nuevo conocimiento en NyN. Además, afecta también las propias evaluaciones de los estudiantes a las cuales están sujetas sus becas, por lo que se advierte la necesidad de que Conacyt sensibilice dicho proceso, particularmente cuando se requiere hacer trabajo experimental o de campo.

Aunado a ello, los procesos administrativos de titulación han sido detenidos o retrasados, posponiendo la titulación de estudiantes de grado y posgrado (por lo menos para dos cohortes), con afectaciones tanto en la eficiencia terminal de los programas docentes, como en la vida profesional del alumnado al no poder transitar hacia el ámbito laboral o a un nivel superior de estudios, entre otras cuestiones por los tiempos que ha tomado la generación de títulos y de liberación de becas.

En síntesis, para los y las encuestadas, la pandemia ha retrasado fuertemente la formación y egreso de estudiantes de grado y posgrado, coincidiendo en $38.9 \%$ en un impacto muy alto y en un $36.6 \%$ alto (figura 9 ). Los impactos de mayor alcance, esto es, en términos del futuro desempeño de los estudiantes formados en la virtualidad, aún están por verse.

Figura 9. Intensidad del retraso percibido en la formación y egreso de estudiantes de grado y posgrado.

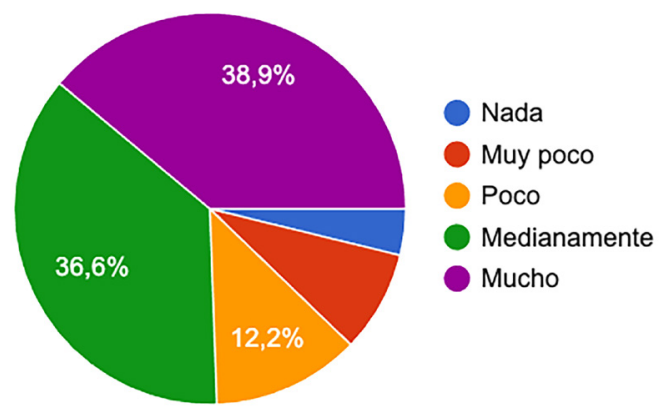

Fuente: Elaboración de los autores.

\section{Reflexiones finales}

El potencial que tiene la NyN para prevenir, diagnosticar y tratar la enfermedad causada por el virus SARS-CoV-2 en sus diversas variantes, pero también otras enfermedades, la hace un área no solo relevante sino estratégica 
para el país, sobre todo dada su dependencia a la importación de insumos, fármacos, instrumental y equipo médico. El apoyo para el avance responsable y bien articulado de la NyN es necesario y la actual situación de crisis sanitaria puede ser una oportunidad para propiciar una demanda de la comunidad que por lo menos tiene más de una década y media. Es también una oportunidad para avanzar aún más en materia de normalización y regulación, así como para propiciar mejores prácticas de investigación, docencia, administración y gestión institucional de manera tal que se pueda cimentar la resiliencia deseable en la actividad científica pero también educativa del país, tanto en el ámbito de las $\mathrm{NyN}$, como en otras áreas.

\section{Referencias}

Adu-Amankwaah, Joseph; Mprah, Richard; Adekunlle, Oluwafemi; Ndzie Noah, Marie et al. 2020. The cardiovascular aspect of COVID-19. Annals of Medicine, 53(1): 227-236. https://doi.org/10.1080/07853890.2020.1861644

Bloom, Jesse; Chan, Yujia A; Baric, Ralph S; Bjorkman, Pamela; Cobey, Sarah et al. 2021. Investigate the origins of COVID-19. Science, 372(6543): 694. https:// doi.org/10.1126/science.abj0016

Chávez-Sandoval, Blanca E.; Flores-Mendoza, Nallely; Chávez-Recio, Auraamellaly; Balderas-López, José A. y García-Franco, Francisco. 2020. Biosíntesis de nanopartículas de oro (AuNPs) y los agentes reductores implicados en el proceso. Mundo Nano. Revista Interdisciplinaria en Nanociencias y Nanotecnología, 14(27): 1e-12e. https:// doi.org/10.22201/ceiich.24485691e.2021.27.69658

Chilazi, Michael; Duffy, Eamon; Thakkar, Aarti y Michos, Erin. 2021. Intermediate and long-term impact of COVID-19 on cardiovascular disease. American College of Cardiology, abril 21. https://www.acc.org/latest-in-cardiology/articles/2021/ 04/21/13/08/intermediate-and-long-term-impact-of-covid-19-on-cardiovascular-disease

Chung, Mina; Zidar, David; Bristow, Michael; Cameron, Scott; Chan, Timothy et al. 2021. COVID-19 and cardiovascular disease. Circulation Research, 128(8): 12141236. https://doi.org/10.1161/CIRCRESAHA.121.317997

CIMAV. 2008. Diagnóstico y prospectiva de la nanotecnología en México. FUNTEC-SE. México. http://www.2006-2012.economia.gob.mx/files/comunidad_negocios/ industria_comercio/Estudios/Diagnostico_y_Prospectiva_Nanotecnologia_ Mexico.pdf

De la Torre, Rocío G. y Betancourt, Israel. 2020. Nanomateriales integrados para el desarrollo de equipo de prevención primaria ante el Covid-19. Mundo Nano. Revista Interdisciplinaria en Nanociencias y Nanotecnología, 14(27): 1e-18e. https:// doi.org/10.22201/ceiich.24485691e.2021.27.69652

Delgado Ramos, Gian Carlo y López García, David. 2020. Las ciudades ante el COVID-19: nuevas direcciones para la investigación urbana y las políticas públicas. PCTUINGSA. CDMX, México. https://zenodo.org/record/3894075/files/Ciudades\%20 ante\%20el\%20COVID-19.pdf?download=1 
Durán Álvarez, Juan C.; Martínez Avelar, Carolina y Mejía Almaguer, Daniel. 2020. El papel de la nanociencia y la nanotecnología en el marco de la pandemia de COVID-19. Mundo Nano. Revista Interdisciplinaria en Nanociencias y Nanotecnología, 14(27): 1e-29e. https://doi.org/10.22201/ceiich.24485691e.2021.27.69647

Florindo, Helena; Kleiner, Ron; Vaskovich-Koubi, Daniella; Acúrcio, Rita et al. 2020. Immune-mediated approaches against COVID-19. Nature Nanotechnology, 15: 630-645. https://doi.org/10.1038/s41565-020-0732-3

FMI. 2021. World economic outlook: Managing divergent recoveries. Fondo Monetario Internacional. https://www.imf.org/en/Publications/WEO/Issues/2021/03/23/worldeconomic-outlook-april-2021

Haider, Najmul; Rothman-Ostrow, Peregrine; Osman, Abdinasir Y.; Arruda, Lia B.; Macfarlane-Berry, Laura et al. 2020. COVID-19 - Zoonosis or emerging infectious disease? Frontiers in Public Health, 8: 596944. https://doi.org/10.3389/ fpubh.2020.596944

IFC. 2020. The impact of COVID-19 on the water and sanitation sector. International Finance Corporation. https://www.ifc.org/wps/wcm/connect/126b1a18-23d9-46f3-beb7047c20885bf6/The+Impact+of+COVID_Water\%26Sanitation_final_web. pdf?MOD=AJPERES\&CVID=ncaG-hA

INEGI. 2021. Producto Interno Bruto de México durante el cuarto trimestre de 2020. Comunicado de prensa No. 157/21. 25 de febrero. https://www.inegi.org.mx/ contenidos/saladeprensa/boletines/2021/pib_pconst/pib_pconst2021_02.pdf

Kollewe, Julia. 2021. From Pfizer to Moderna: who's making billions from COVID-19 vaccines? The Guardian, 6 de marzo. https://www.theguardian.com/ business/2021/mar/06/from-pfizer-to-moderna-whos-making-billions-fromcovid-vaccines

Lammers, Twan; Marios Sofias, Alexandros; Van der Meel, Roy; Schiffelers, Raymond et al. 2020. Dexamethasone nanomedicines for COVID-19. Nature Nanotechnology, 15: 622-624. https://doi.org/10.1038/s41565-020-0752-z

Lancet. 2021. Urgent needs of low-income and middle-income countries for COVID-19 vaccines and therapeutics. The Lancet, 397(10274): 562-564. https:// doi.org/10.1016/S0140-6736(21)00242-7

Marc, Malgorzata A.; Domínguez-Álvarez, Enrique y Gamazo, Carlos. 2015. Nucleic acid vaccination strategies against infectious diseases. Expert Opinion on Drug Delivery, 12: 1851-1865. https://doi.org/10.1517/17425247.2015.1077559

MHRA. 2020. Public Assessment Report. Authorisation for Temporary Supply. Medicines \& Healthcare products Regulatory Agency. Reino Unido. https://assets.publishing.service.gov.uk/government/uploads/system/uploads/attachment_data/ file/944544/COVID19_mRNA_Vaccine_BNT162b2_UKPAR_PFIZER_ BIONTECH_15Dec2020.pdf

Milane, Lara y Amiji, Mansoor. 2021. Clinical approval of nanotechnology-based SARS-CoV-2 mRNA vaccines: impact on translational nanomedicine. Drug Delivery and Translational Research, https://doi.org/10.1007/s13346-021-00911-y

Navarrete, Alejandro y Treviño, Ricardo. 2020. El ventilador vs COVID-19 que unió a empresas, gobierno y universidad. Tecnológico de Monterrey. 6 de agosto. 
https://tec.mx/es/noticias/nacional/salud/el-ventilador-vs-covid-19-queunio-empresas-gobierno-y-universidad

NBSC. 2021. National economy recovered steadily in 2020 with main goals accomplished better than expectation. National Bureau of Statistics of China. 18 de enero. http://www.stats.gov.cn/english/PressRelease/202101/t20210118_1812432. html\#: : text=According\%20to\%20preliminary\%20estimates\%2C\%20 the,last\%20year\%20at\%20comparable\%20prices.

Neidleman, Jason; Luo, Xiaoyu; McGregor, Matthew; Xie, Guorui; Murray, Victoria et al. 2021. mRNA vaccines-induced SARS-CoV-2 specific T cells recognize B.I.I7 and B.I.35I variants but differ in longevity and homing properties depending on prior infection status. bioRxiv. https://doi.org/10.1101/2021.05.12.443888

OMS. 2021-A. WHO-convened global study of origins of SARS-CoV-2: China Part. 14 de enero. https://www.who.int/publications/i/item/who-convened-global-studyof-origins-of-sars-cov-2-china-part

OMS. 2021-B. WHO urges countries to build a fairer, healthier world post-COVID-19. 6 de abril. https://www.who.int/news/item/06-04-2021-who-urges-countriesto-build-a-fairer-healthier-world-post-covid-19

Pardi, Norbert; Hogan, Michael; Porter, Frederick y Weissman, Drew. 2018. mRNA vaccines - a new era in vaccinology. Nature Reviews Drug Discovery, 17: 261279. https://doi.org/10.1038/nrd.2017.243

Pramanik, Avijit; Gao, Ye; Patibandla, Shamily; Mitra, Dipanwita; McCandless, Martin et al. 2021. The rapid diagnosis and effective inhibition of coronavirus using spike antibody attached gold nanoparticles. Nanoscale Advances, 3: 1588-1596. https://doi.org/10.1039/D0NA01007C

Rabiee, Navid; Bagherzadeh, Mojtaba; Ghasemi, Amir; Zare, Hossein; Ahmadi, Sepideh et al. 2020. Point-of-Use Rapid Detection of SARS-CoV-2: Nanotechnology-Enabled Solutions for the COVID-19 Pandemic. International Journal of Molecular Sciences, 21(4): 5126. https://doi.org/10.3390/ijms21145126

Rashidzadeh, Hamid; Danafar, Hossein; Rahimi, Hossein; Mozafari, Faezeh; Salehiabar, Marziyeh et al. 2021. Nanotechnology against the novel coronavirus: diagnosis, treatment, therapy and future perspectives. Nanomedicine, 16(6). https://doi.org/10.2217/nnm-2020-0441

Rodríguez García, Arturo. El CONACYT presenta dos ventiladores para pacientes COVID de diseño mexicano. Proceso, 14 de julio. https://www.proceso.com.mx/ nacional/2020/7/14/el-conacyt-presenta-dos-ventiladores-para-pacientes-covid-de-diseno-mexicano-246122.html

Seo, Giwan; Lee, Geonhee; Kim, Mi J.; Baek, Seung-Hwa; Choi, Minsuk et al. 2020. Rapid detection of COVID-19 causative virus (SARS-CoV-2) in human nasopharyngeal swab specimens using field-effect transistor-based biosensor. ACS Nano, 14(4): 5135-5142. https://doi.org/10.1021/acsnano.0c02823

Talebian, Sepehr; Wallace, Gordon; Schroeder, Avi; Stellacci, Francesco y Conde Joao. 2020. Nanotechnology-based disinfectants and sensors for SARS-CoV-2. Nature Nanotechnology, 15: 618-621. https://doi.org/10.1038/s41565-020-0751-0

UAM. 2018. Nano-molécula diseñada por egresada de la UAM, útil en el sector salud 
y en el cuidado personal. Boletines UAM, 209. 27 de marzo. http://www.comunicacionsocial.uam.mx/boletinesuam/209-18.html

UNWTO. 2020. The impact of COVID19 on tourism. World Tourism Organization. https:// webunwto.s3.eu-west-1.amazonaws.com/s3fs-public/2020-08/UN-Tourism-Policy-Brief-Visuals.pdf

Vadlamani, Bhaskar; Uppal, Timsy; Verma, Subhash y Misra, Mano. 2020. Functionalized $\mathrm{TiO}_{2}$ nanotube-based electrochemical biosensor for rapid detection of SARS-CoV-2. Sensors, 20(20): 5871. https://doi.org/10.3390/s20205871

Velikova, Tsvetelina y Goergiev, Tsvetoslav. 2021. SARS-CoV-2 vaccines and autoimmune diseases amidst the COVID-19 crisis. Rheumatology International, 41: 509-518. https://doi.org/10.1007/s00296-021-04792-9

Ventura, Bartolomeo; Cennamo, Michele; Minopoli, Antonio; Campanile, Raffaele et al. 2020. Colorimetric test for fast detection of SARS-CoV-2 in nasal and throat swabs. ACS Sensors, 5(10): 3043-3048. https://doi.org/10.1021/acssensors.0c01742 